\title{
Chapter 13 \\ LEAP-UCD-2017 Centrifuge Test at University of California, Davis
}

\author{
Trevor J. Carey, Nicholas Stone, Masoud Hajialilue Bonab, \\ and Bruce L. Kutter
}

\begin{abstract}
Three centrifuge experiments were performed at the University of California, Davis, for LEAP-UCD-2017. LEAP is a collaborative effort to assess repeatability of centrifuge test results and to provide data for the validation of numerical models used to predict the effects of liquefaction. The model configuration used the same geometry as the LEAP-GWU-2015 exercise: a submerged slope of Ottawa F-65 sand inclined at 5 degrees in a rigid container. This paper focuses on presenting results from the two destructive ground motions from each of the three centrifuge models. The effect of each destructive ground motion is evaluated by accelerometer recordings, pore pressure response, and lateral deformation of the soil surface. New techniques were implemented for measuring liquefaction-induced lateral deformations using five GoPro cameras and GEO-PIV software. The methods for measuring the achieved density of the as-built model are also discussed.
\end{abstract}

\subsection{Introduction}

The current phase of LEAP, LEAP-UCD-2017, involved centrifuge experiments conducted at nine different research facilities, including the University of California, Davis (UCD). The experiment, similar to LEAP-GWU-2015 (Kutter et al. 2017), consisted of a submerged clean sand sloped at 5 degrees, subjected to a $1 \mathrm{~Hz}$ ramped sine wave ground motion inputted at the base of a rigid model container. Three experiments were performed on the $1 \mathrm{~m}$ radius Schaevitz centrifuge at the Center for Geotechnical Modeling. The $1 \mathrm{~m}$ centrifuge performs shaking in the circumferential direction of the centrifuge. Detailed specifications by Kutter et al. (2019a) were provided to facilitate replicability among the different centrifuge facilities.

Discussed herein are the specifications that pertained specifically to the UCD

T. J. Carey $(\bowtie) \cdot$ N. Stone $\cdot$ B. L. Kutter

Department of Civil and Environmental Engineering, University of California, Davis, CA, USA

e-mail: tjcarey@ucdavis.edu

M. H. Bonab

Department of Civil Engineering, University of Tabriz, Tabriz, Iran

(C) The Author(s) 2020

B. Kutter et al. (eds.), Model Tests and Numerical Simulations of Liquefaction

and Lateral Spreading, https://doi.org/10.1007/978-3-030-22818-7_13 
experiments and deviations from the specifications, both intended and unintended. The implementation of a new technique for measuring slope deformations during strong shaking is discussed and the process for measuring the achieved dry density of a constructed model and saturation protocol are detailed. Kutter et al. (2019b) provide a detailed comparison of all centrifuge experiments from the nine participating facilities, including the results presented herein.

\subsection{UC Davis Test Specific Information}

\subsubsection{Description of the Model and Instrumentation}

The container dimensions for the UCD experiments are $457.2 \mathrm{~mm}(\mathrm{~L}) \times 279.4 \mathrm{~mm}$ $(\mathrm{W}) \times 177.8 \mathrm{~mm}(\mathrm{H})$. The $457.2 \mathrm{~mm}$ length of the container was obtained by placing 25.4-mm-thick plastic plates on each end wall of the rigid container. The plastic plates were needed to ensure that the soil would remain completely submerged at $1 \mathrm{~g}$ without overtopping when the water surface curved during spinning. Figure 13.1 details the test geometry, sensor locations, and PVC blocks in model scale. Note that Carey et al. (2017) used a different model container and spacer configuration for LEAP-GWU-2015.

A flat soil surface in a centrifuge represents a hill in prototype. To model a flat surface in prototype, the model surface should be curved with the same radius as the centrifuge. A 5-degree slope from the normal of the radial g-field would have a varying radius along the slope of the model surface, which is described theoretically by a log-spiral. Carey et al. 2017 showed that rotating a circular arc by 5 degrees is a reasonable approximation for the log-spiral surface.

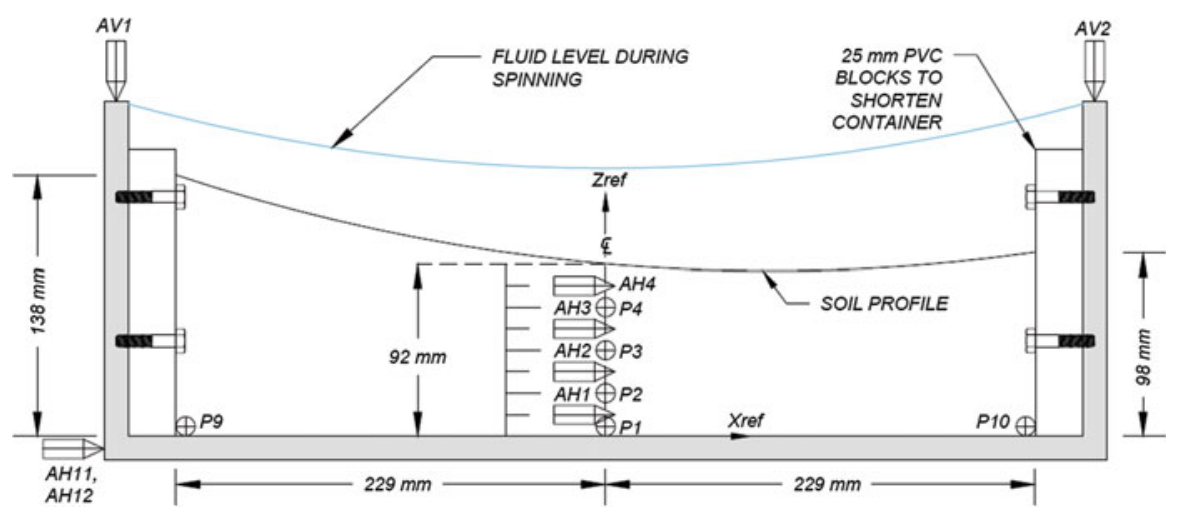

Fig. 13.1 Model geometry and sensor layout (dimensions in model scale) 
Fig. 13.2 Wooden template and vacuum tool used to excavate sand to produce the log-spiral surface

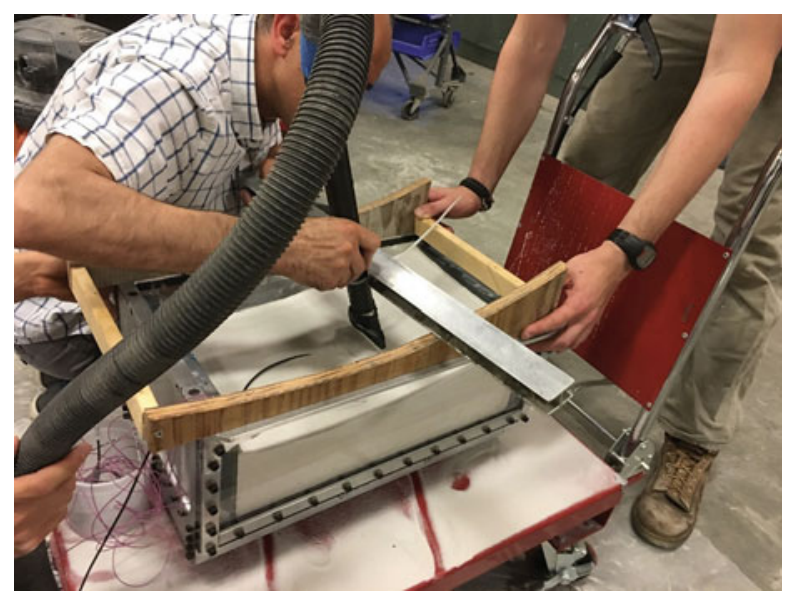

Figure 13.2 shows the wooden template fabricated to approximate the log-spiral surface of the model. Sand was first pluviated to a depth above the desired final curved surface. Using a depth gauge, the amount of soil to remove was roughly calculated by measuring the depth of the model at the ends and midpoint. Excess soil was removed by vacuuming across and then down the slope. The final depths of the model were verified at each quarter point. With this method, the final surface depth could be constructed to $\pm 1 \mathrm{~mm}$ of the specified height.

\subsubsection{Sensors}

The number of sensors placed in the model was limited by the capacity of the data acquisition system; therefore, only the required pore pressure transducers (P1, P2, P3, P4, P9, and P10) and accelerometers (AH1, AH2, AH3, AH4, AH11, AH12, AV1, AV2) were included.

\subsubsection{Scaling Laws}

The scaling laws for LEAP-UCD-2017 are provided by Kutter et al. (2019a). The length scale factor $L^{*}$ is defined as $L^{*}=L_{\mathrm{MODEL}} / L_{\mathrm{PROTOTYPE}}=(0.457 \mathrm{~m}) /$ $(20 \mathrm{~m})=1 / 43.75$. Using the conventional centrifuge scaling law for gravity, $g^{*}=1 / L^{*}=43.75$. An angular velocity of $194 \mathrm{RPM}$ was determined to produce $g^{*}=43.75$ at the effective radius of $1.033 \mathrm{~m}$ (the radius to $1 / 3$ of the depth of soil). 


\subsection{Test Results}

UCD performed three experiments with a target dry density of $1651 \mathrm{~kg} / \mathrm{m}^{3}$. The measured dry densities of the three models ranged from 1648 to $1665 \mathrm{~kg} / \mathrm{m}^{3}$. Densities were calculated by the measurement of mass and volume. A more detailed explanation of the method used to measure the volume of each model is described later in this paper. Each specimen used the standard pluviator, which consisted of a No. 16 sieve with three slots (Kutter et al. 2019a) to place the soil. To provide the best likelihood to obtain the target density, the drop height from the standard pluviator to the model surface was adjusted before each experiment, based on the previous model's measured density. The calculated achieved density does, however, seem to be affected by unaccounted for factors such as humidity, temperature, personal habits and uncertain measurements of mass and volume.

\subsubsection{Achieved Ground Motions}

Recognizing the achieved base motion will contain additional frequencies superimposed on the $1 \mathrm{~Hz}$ motion, Kutter et al. (2019a) introduced PGA effective $_{\text {as }}$ a ground motion intensity parameter, where $\mathrm{PGA}_{\text {effective }}=\mathrm{PGA}_{1 \mathrm{~Hz}}+1 / 2 \mathrm{PGA}_{\text {highfreq }}$. The PGA of the $1 \mathrm{~Hz}$ and high-frequency components are found using a filtering scheme described by Kutter et al. (2019a). Table 13.1 lists the sequence of shaking, and corresponding effective PGAs for each of the three models. Additional nondestructive motions were performed prior to and following each destructive motion, consisting of a series of square pulses with a PGA less than $0.04 \mathrm{~g}$ in an attempt to characterize the specimen shear-wave velocity. These motions were considered to be nondestructive due to their low magnitude and high-frequency content; however, these nondestructive motions have not been fully processed. The raw data from the nondestructive motions is archived in NHERI DesignSafe with the processed data of the destructive motions.

Table 13.1 Ground motion PGAs for the three UCD experiments

\begin{tabular}{l|l|l|l|l|l|l|l|l}
\hline & \multicolumn{3}{|l}{ Destructive motion 1 } & \multicolumn{3}{l}{ Destructive motion 2 } \\
\hline & $\begin{array}{l}\mathrm{PGA}_{\text {raw }} \\
(\mathrm{g})\end{array}$ & $\begin{array}{l}\mathrm{PGA}_{\text {eff }} \\
(\mathrm{g})\end{array}$ & $\begin{array}{l}\mathrm{PGA}_{1 \mathrm{~Hz}} \\
(\mathrm{~g})\end{array}$ & $\begin{array}{l}\mathrm{PGA}_{\mathrm{HF}} \\
(\mathrm{g})\end{array}$ & $\begin{array}{l}\mathrm{PGA}_{\text {raw }} \\
(\mathrm{g})\end{array}$ & $\begin{array}{l}\mathrm{PGA}_{\text {eff }} \\
(\mathrm{g})\end{array}$ & $\begin{array}{l}\mathrm{PGA}_{1 \mathrm{~Hz}} \\
(\mathrm{~g})\end{array}$ & $\begin{array}{l}\mathrm{PGA}_{\mathrm{HF}} \\
(\mathrm{g})\end{array}$ \\
\hline UCD1 & 0.165 & 0.149 & 0.119 & 0.060 & 0.378 & 0.296 & 0.214 & 0.163 \\
\hline UCD2 & 0.339 & 0.210 & 0.149 & 0.122 & 0.248 & 0.220 & 0.179 & 0.083 \\
\hline UCD3 & 0.192 & 0.183 & 0.134 & 0.099 & 0.180 & 0.172 & 0.132 & 0.081 \\
\hline
\end{tabular}




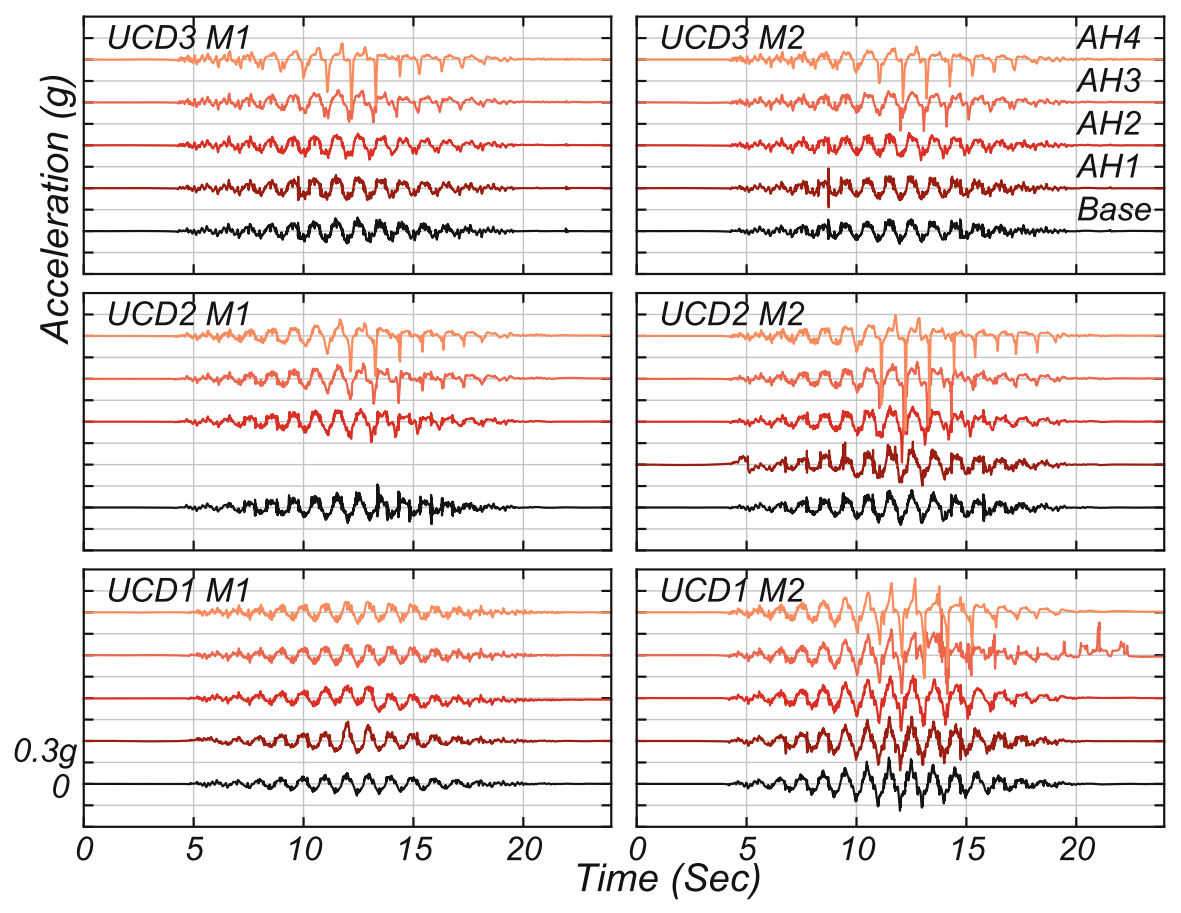

Fig. 13.3 Horizontal acceleration time histories for UCD1, UCD2, and UCD3 (two destructive motions each)

\subsubsection{Accelerometer Records During Destructive Motions}

The acceleration time histories of the input base motion and the four central horizontal accelerometers (AH1, AH2, AH3, and AH4) are reported in Fig. 13.3. The input base motion is taken as the average of the accelerations measured by AH11 and AH12.

- UCD1

- Destructive motion 1: The surface accelerometers recorded an almost identical motion as the base input motion. Both motions appear to remain in phase with one another, implying the soil moved nearly as a rigid mass, with small shear strains, and no evidence of liquefaction.

- Destructive motion 2: The second motion, M2, was the strongest of all the destructive motions. Initially, the central array sensors are in-phase with the input motion. As liquefaction occurs, beginning at the shallowest sensors first, dilation spikes, characterized as sharp, sudden accelerations, occur. The magnitudes of the spikes decrease with depth since the severity of liquefaction is decreasing with depth. 


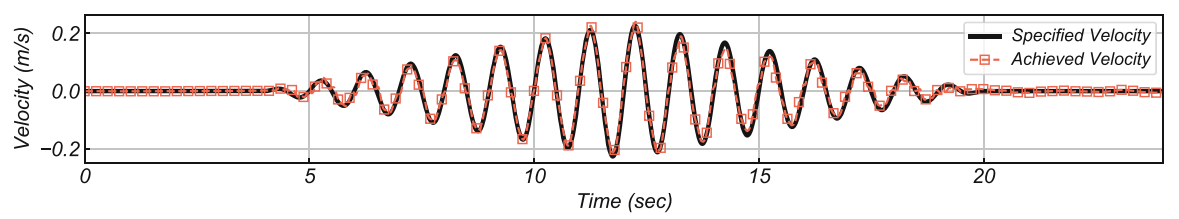

Fig. 13.4 Specified versus achieved velocity for UCD2 destructive motion 1

\section{- UCD2}

- Destructive motion 1: The input base motion had high-frequency components superimposed on the $1 \mathrm{~Hz}$ signal, especially from 12-16 seconds. It is believed that this behavior may have been caused by insufficient pressure in the supply accumulator for the hydraulic shaker. When the achieved velocity of the base motion (found by integrating the achieved base motion) is compared with the specified velocity in Fig. 13.4, good agreement is observed. This indicates the superimposed high-frequency accelerations had minimal effect on the base input motion velocity.

- Destructive motion 2: The input motion contained a larger $(0.18 \mathrm{~g}) 1 \mathrm{~Hz}$ component than M1 (0.15 g), but M2 had a smaller high-frequency component $(0.083 \mathrm{~g})$ compared to M1 (0.122 g) (see Table 13.1). The reduction of the high-frequency components was partly from increasing the hydraulic pressure to the accumulator. The dilation spikes for M2 have larger magnitudes than the spikes in M1, indicating more severe liquefaction in M2 than in M1. This also indicates that the severity of liquefaction dilation is more sensitive to the low-frequency components of the input motion.

\section{- $\mathrm{UCD} 3$}

- Destructive motions 1 and 2: The input motions for M1 and M2 are nearly repeated in terms of magnitude, frequency content, and duration. Similar dilation spikes occur in both motions, but differences in the magnitudes can be observed. The spikes for AH4 are larger during M1 compared to M2, but the AH3 spikes are larger during M2. This could indicate localized zones of loose and dense material.

\subsubsection{Excess Pore Pressures}

The excess pore pressures of the central array generated during strong shaking are reported in Fig. 13.5. P1 is located on the base of the container, at roughly a depth of $4 \mathrm{~m}$, and P4 is at the surface, at $1 \mathrm{~m}$ depth. The initial vertical effective stress $\left(\sigma^{\prime}\right)$ prior to shaking is approximately $40,30,20$, and $10 \mathrm{kPa}$ for $\mathrm{P} 1, \mathrm{P} 2, \mathrm{P} 3$, and $\mathrm{P} 4$, respectively. 


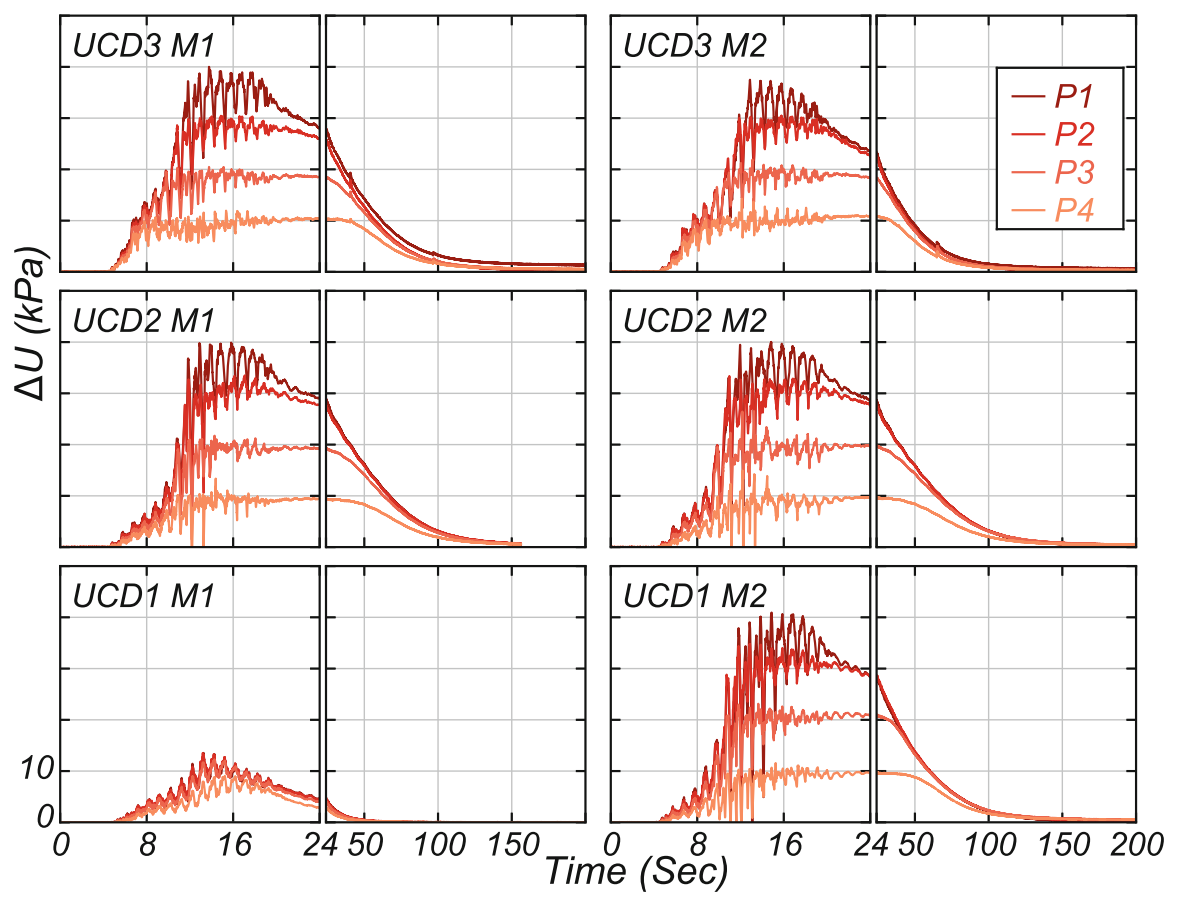

Fig. 13.5 Central array excess pore pressures for UCD1, UCD2, and UCD3 (two destructive motions each)

\section{- UCD1}

- Destructive motion 1: Similar to observations made of the acceleration response, the pore pressure response during M1 does not indicate that liquefaction occurred. The excess pore pressure ratios for P1 through P4 were 34\%, $45 \%, 62 \%$, and $90 \%$. Once the magnitude of the base motion started to decrease at approximately $12 \mathrm{~s}$, excess pore pressures immediately began to dissipate, indicating that at the depths of the PPTs the pore pressure never reached a constant state equal to their respective vertical effective stresses.

- Destructive motion 2: The generation of excess pore pressure starts slow but as the magnitude of shaking increases each transducer reaches its initial vertical effective stress. Deliquefaction shockwaves, manifested as a sudden drop in porewater pressure due to the sudden dilation of a liquefied soil, are observed after the magnitude of the excess pore pressure is equal to the initial vertical effective stress (Kutter and Wilson 1999). Once shaking intensity decreases, pore pressures at P1, the deepest sensor, begin to dissipate first. Dissipation is followed then by the shallower sensors. 


\section{- $\mathrm{UCD} 2$}

- Destructive motions 1 and 2: The effect of the high-frequency component of the base motion is not easily seen in the pore pressure response for M1 and M2, indicating that the overall effect to the model performance is minimal. The stronger $1 \mathrm{~Hz}$ component in M2 is noticeable with the quicker accumulation of excess pore pressures for P1 and P2, compared with M1.

\section{- UCD3}

- Destructive motions 1 and 2: Both M1 and M2 led to the fastest generation of excess pore pressure of all the tests conducted at UCD. At roughly $8 \mathrm{~s}$ the excess pore pressure ratio for $\mathrm{P} 4$ is already close to $100 \%$; the other sensors indicated liquefaction later. Following shaking, the excess pore pressures were not maintained for the same duration when compared with the other experiments. The dissipation time for P1 and P2 after M2 was noticeably faster than after M1. This is possibly due to densification during M1.

Figure 13.6 shows the vertical component of acceleration for each destructive motion. The top acceleration trace is AV1 and the bottom is AV2. A fourth order band-pass filter with corner frequencies of $0.3-3 \mathrm{~Hz}$ was used to remove high-

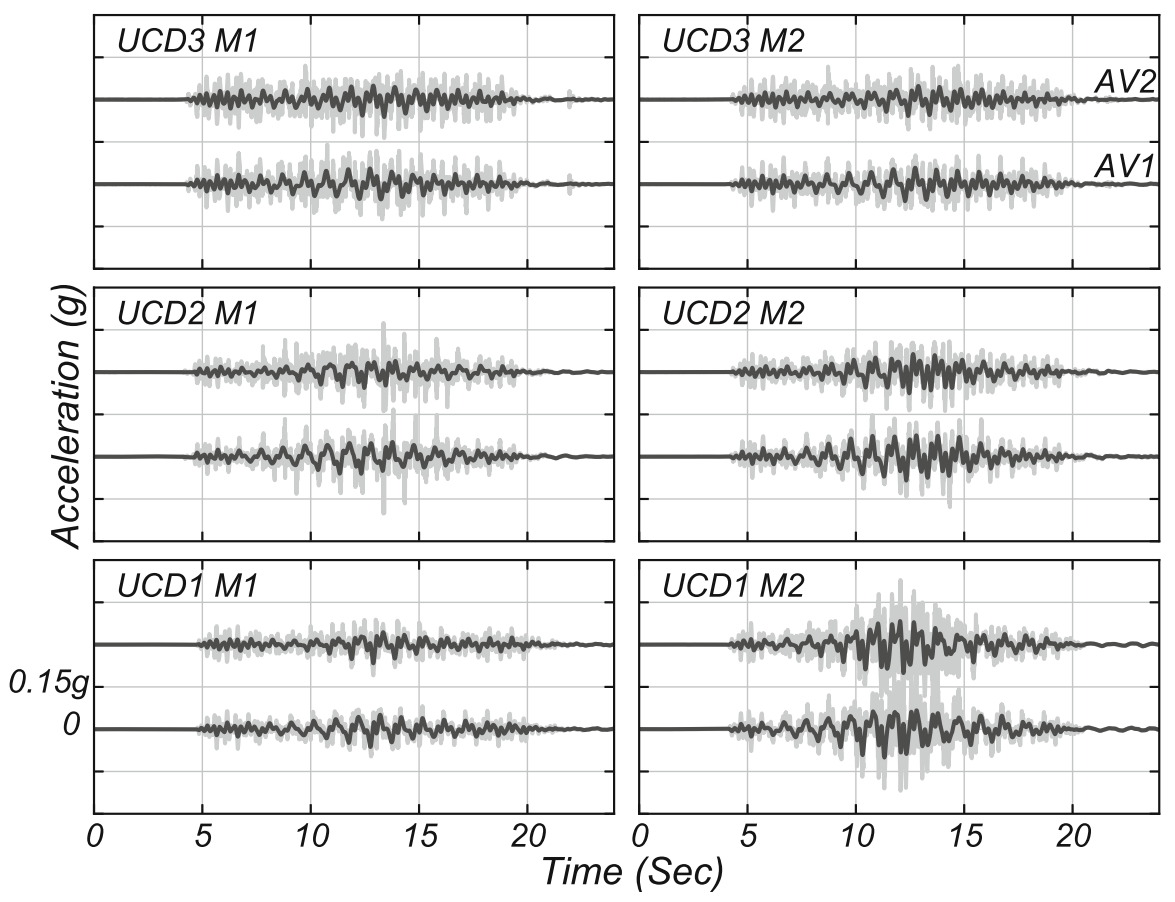

Fig. 13.6 Filtered and non-filtered vertical acceleration time histories for UCD1, UCD2, and UCD3 (two destructive motions each) 


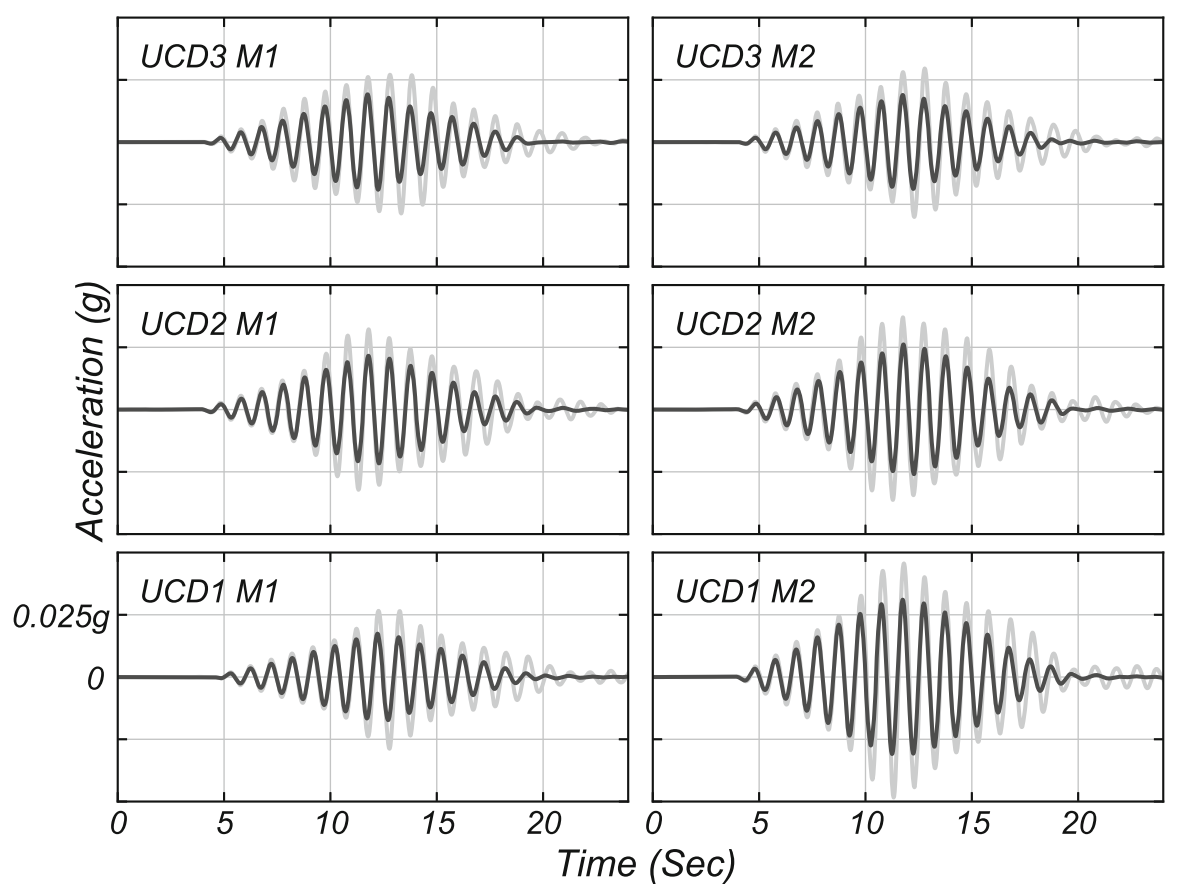

Fig. 13.7 Filtered $1 \mathrm{~Hz}$ vertical acceleration (gray) and Coriolis acceleration calculated from the container input motion (black) for UCD1, UCD2, and UCD3 (two destructive motions each)

frequency noise, similar to that described by Kutter et al. 2017. The gray trace is the unfiltered response and the black trace shows the filtered response. Little to no phase shift is observed between the $1 \mathrm{~Hz}$ component measured at AV1 and AV2, but the higher-frequency components do appear to be out of phase. This suggests that rocking occurs at a $3 \mathrm{~Hz}$ or higher frequencies. The $1 \mathrm{~Hz}$ component of vertical acceleration may be the result of the Coriolis acceleration. The Coriolis acceleration is expressed as $\bar{a}_{\text {cor }}=2 \Phi v_{\text {rel }}$, where $\omega$ is rotational angular velocity of the centrifuge and $v_{\text {rel }}$ is the velocity of the container input motion. For example, the Coriolis acceleration for the UCD experiments $(\omega=20.36 \mathrm{rads} / \mathrm{s})$ with a $0.15 \mathrm{~m} / \mathrm{s} 1 \mathrm{~Hz}$ component of velocity is $0.015 \mathrm{~g}$. In Fig. 13.7 the Coriolis acceleration calculated from the container input motion shown as black and the average vertical acceleration from sensors AV1/AV2 in gray are compared. The $1 \mathrm{~Hz}$ vertical acceleration and $1 \mathrm{~Hz}$ velocity were obtained with a notch filter with corner frequencies of 0.5 and $1.5 \mathrm{~Hz}$. The measured $1 \mathrm{~Hz}$ vertical acceleration in Fig. 13.7 is systematically larger than the calculated Coriolis acceleration. Therefore, the recorded vertical accelerations shown in Figs. 13.6 and 13.7 are amplified from the Coriolis acceleration shown in Fig. 13.7. 


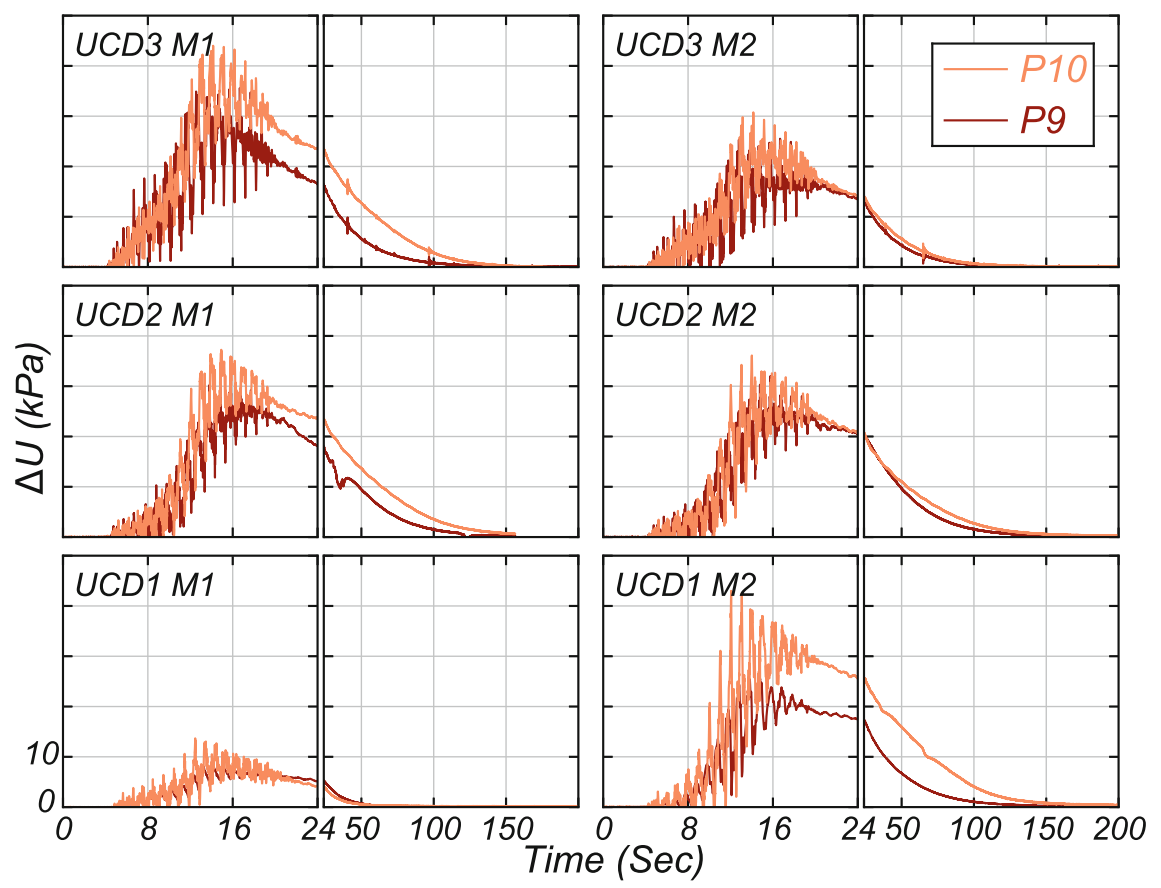

Fig. 13.8 Bottom centerline pore pressure responses during UCD1, UCD2, and UCD3 (two destructive motions each)

Figure 13.8 shows the pore pressure transducer response of $\mathrm{P} 9$ and $\mathrm{P} 10$ for all destructive motions. Both $\mathrm{P} 9$ and $\mathrm{P} 10$ are located at the base of the container, but P9 has greater initial vertical effective stress since it is on the upslope side of the model. Suggested by Kutter et al. (2017), these two sensors are positioned to be sensitive to tilt of the model container that may occur because of friction generated when the bucket swings up. They also serve to confirm that the water level is constant; the return of the pore pressures to $\Delta \mathrm{u}=0$ in Fig. 13.8 indicates that the water table before and after shaking remained unchanged in the UCD experiments. Liquefaction at either of these sensors is difficult to achieve since no upward seepage of pore pressure can occur beneath the base of the container, and the rigid walls at the base of the container constrain against the development of shear strains. The oscillation of total stress along the ends of the container could be causing the dynamic oscillation in porewater pressure apparent in Fig. 13.8. The total stress at P9 and P10 is about $100 \mathrm{kPa}$; thus $a \pm 0.05 \mathrm{~g}$ vertical acceleration at the ends of the container (e.g., the low-frequency components in Fig. 13.6) could produce a cyclic total vertical stress of about $\pm 5 \mathrm{kPa}$, which is consistent with the low-frequency $(\sim 1 \mathrm{~Hz})$ component of the pore pressures at $\mathrm{P} 9$ and $\mathrm{P} 10$. 
Fig. 13.9 Cone penetration tests for UCD1, UCD2, and UCD3, before and after each destructive motion

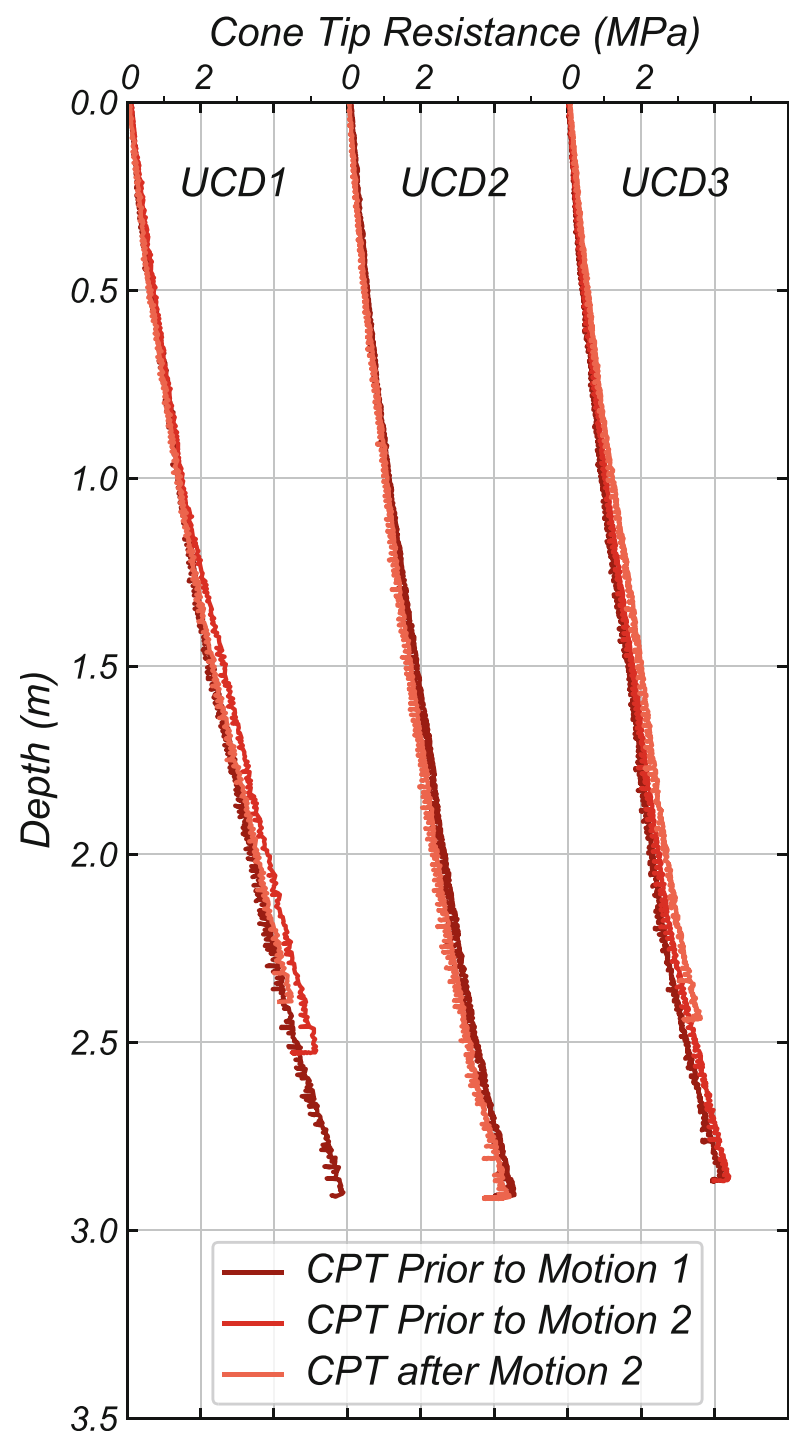

\subsubsection{Cone Penetration Tests}

Three CPT soundings were performed during each experiment using the CPT specifically designed for the LEAP project (Carey et al. 2018a). A sounding was performed prior to destructive motions 1 and 2 and after destructive motion 2. CPT soundings were used as an independent check of the density and state of the specimen prior to each destructive motion for numerical prediction of the experiment. Figure 13.9 shows the response obtained from each CPT. Overall, little change in cone tip resistance $\left(q_{\mathrm{c}}\right)$ was observed between destructive motions. 
UCD3 shows an approximate 5\% increase in $q_{\mathrm{c}}$ at $2 \mathrm{~m}$ depth between M1 and M2. UCD2 shows very small (less than $2 \%$ ) decrease in $q_{\mathrm{c}}$ at $2 \mathrm{~m}$ depth between M1 and M2. $q_{\mathrm{c}}$ increases by approximate $5 \%$ at $2 \mathrm{~m}$ depth after UCD1 M1, but an approximate $5 \%$ decrease in $q_{\mathrm{c}}$ at $2 \mathrm{~m}$ depth after M2. As previously discussed, UCD1 M1 did not liquefy the model; therefore, the variation in the cone profiles may be attributed to a combination of effects of spatial variability, lateral stresses, and soil density.

\subsubsection{Surface Marker Surveys}

Surface markers were placed in three longitudinal arrays, with each array consisting of 6 markers, for a total of 18 surface markers. Surface marker locations were recorded prior to and following each destructive motion to track lateral and vertical deformations. Figures 13.10, 13.11, and 13.12 show the displacement path of the central array of surface markers during the first destructive motion of UCD1, UCD2, and UCD3, respectively. The surface markers for UCD2 M1 and UCD3 M1 displaced with typical deformation tendencies of a sloping liquefiable deposit. All surface markers moved downslope; upslope markers tended to settle, while downslope markers tended to heave. Surface markers in UCD1 M1 underwent little deformation. Not seen in Figs. 13.10, 13.11, and 13.12, the outer arrays of surface markers followed similar deformation patterns as the central arrays.

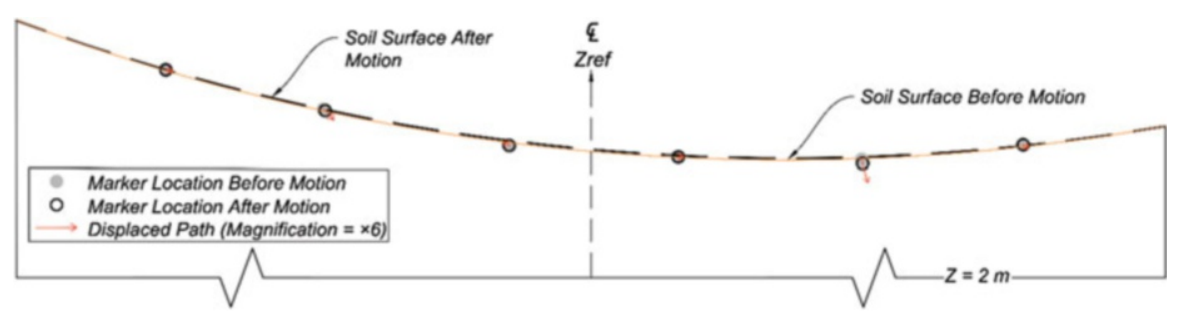

Fig. 13.10 Surface marker displacement from hand measurements during UCD1 M1

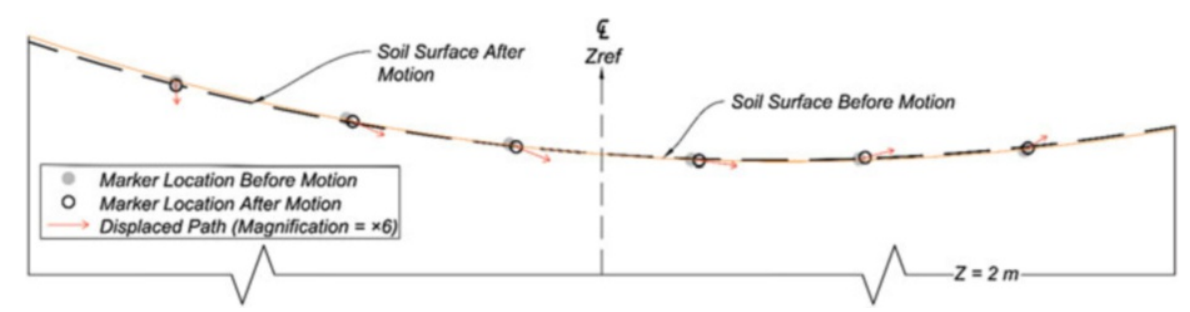

Fig. 13.11 Surface marker displacement from hand measurements during UCD2 M1 


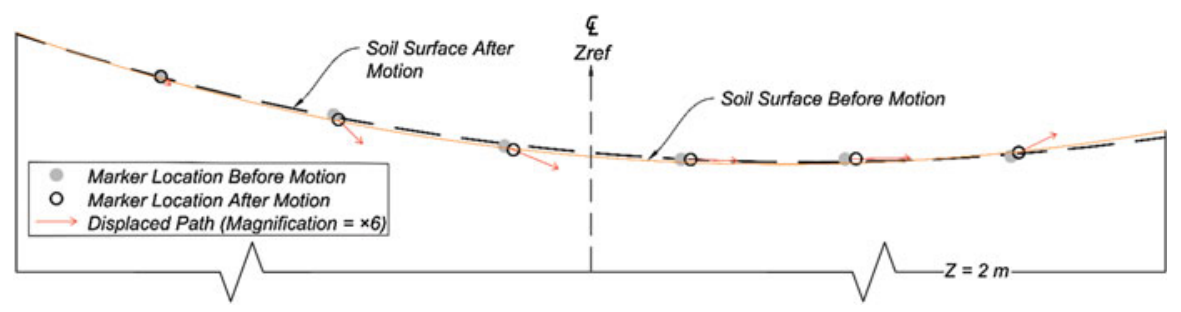

Fig. 13.12 Surface marker displacement from hand measurements during UCD3 M1

Fig. 13.13 UCD2 surface marker array, with downslope center markers 4 and 5 offset to accommodate the wire from AH4 (image generated by superimposing images together from five cameras)

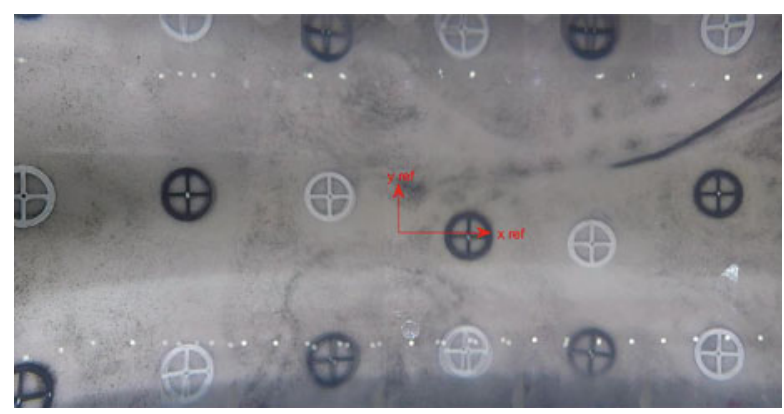

\subsection{Nonconformities with Specifications}

As discussed, each model contained three longitudinal arrays of six surface markers, for a total of 18 markers. In UCD2 and UCD3, the top accelerometer (AH4) cable was too close to the surface, making it impossible to embed the lower slope centerline surface markers without disturbing the sensor. In UCD2, the fourth and fifth surface markers from the upslope end of the model were offset from the centerline by $25 \mathrm{~mm}$. In UCD3, the fourth, fifth, and sixth surface markers from the upslope end of the model were offset from the centerline by $25 \mathrm{~mm}$. Figure 13.13 shows an example of the $25 \mathrm{~mm}$ offset from UCD2.

Several nondestructive motions were performed prior to and following each destructive motion as discussed earlier. For UCD1, additional destructive motions were applied after M2 for the purposes of calibrating shaker command motions for subsequent model tests; these motions are not analyzed in the present paper.

\subsection{Advancements in Centrifuge Testing}

LVDTs have been used previously to measure displacement of liquefied geosystems, but others (Fiegel and Kutter 1994) have noted the measurements may be unreliable during liquefaction. For LEAP-UCD-2017, a new procedure for tracking slope 


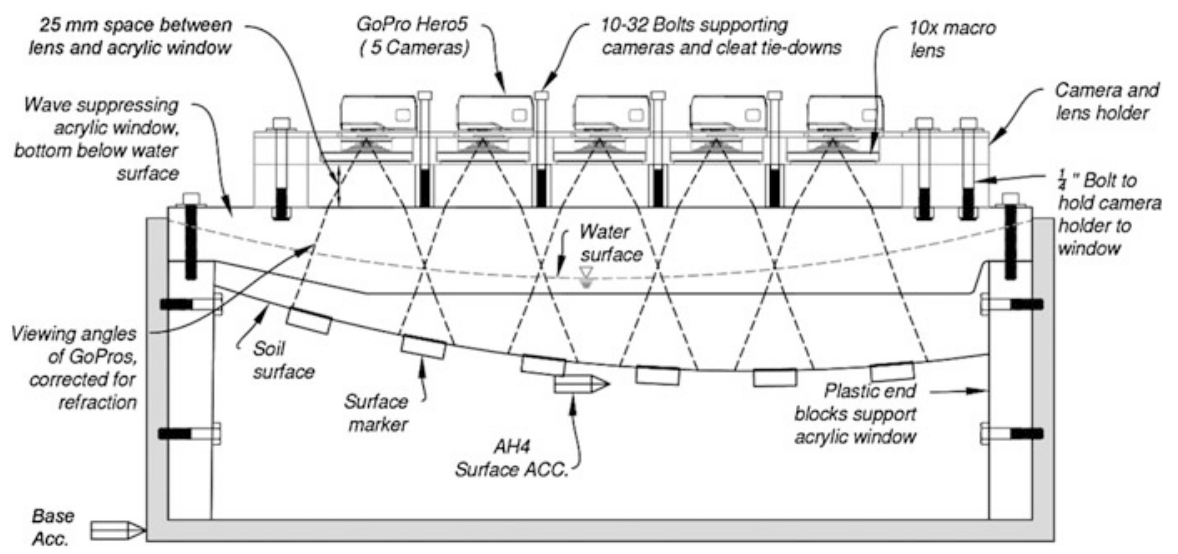

Fig. 13.14 Profile view of centrifuge container with acrylic glass window and mounted GoPro cameras viewing through wide angle lenses

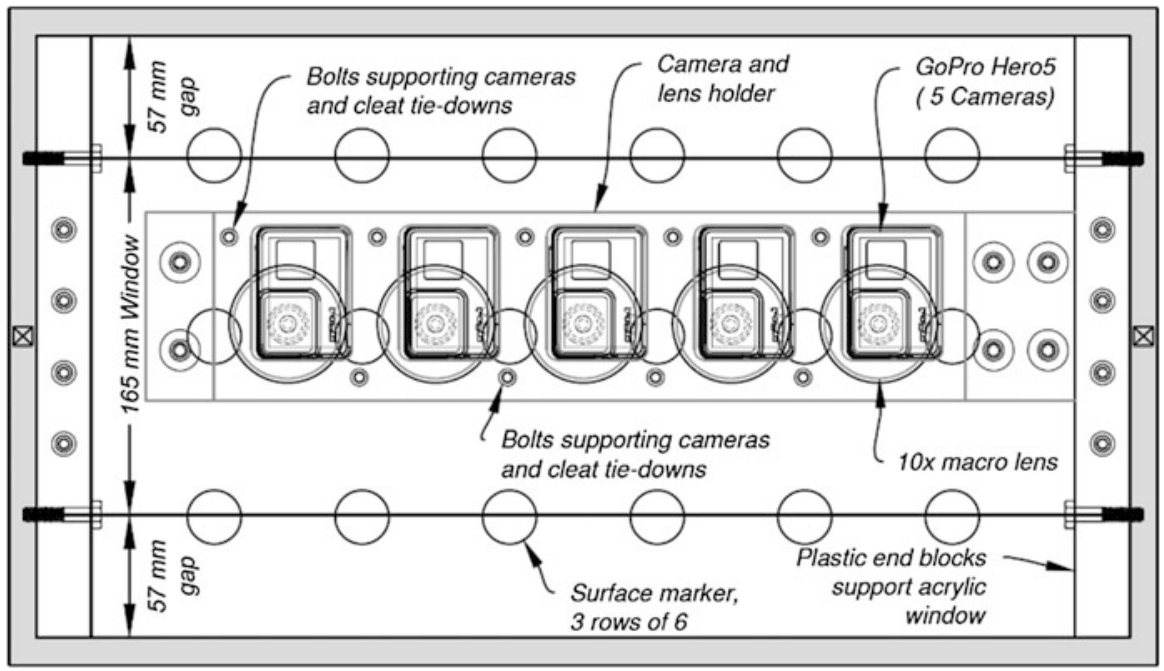

Fig. 13.15 Top view of centrifuge container with acrylic glass window and mounted GoPro cameras viewing through wide angle lenses

displacements of a centrifuge model using a wave suppressing window, GoPro cameras, and GEO-PIV software (Carey et al. 2018b) was developed. The bottom of the wave suppressing window was located beneath the fluid surface to reduce distortion due to water surface waves, like a glass-bottomed boat as shown in Figs. 13.14 and 13.15 . Note that the $57 \mathrm{~mm}$ gap between the window and the side walls of the container allowed for a free water surface and ensured that window vibrations could not cause significant water pressure oscillations. Five GoPro 

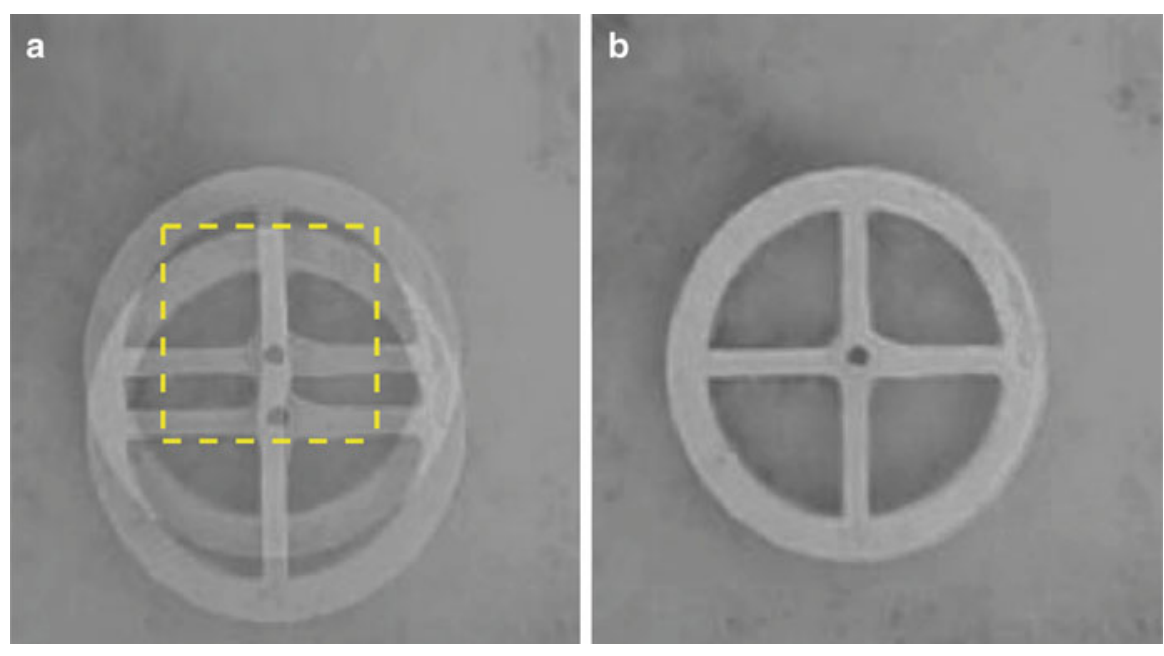

Fig. 13.16 Validation of GEO-PIV using manual image processing in MATLAB, (a) location of a surface marker prior to and following shaking overlaid shown with a typical PIV patch. (b) the image recorded prior to shaking is shifted until a clear image of the surface marker is obtained

cameras were secured in a camera holder mounted above the window. $10 \times$ macro lenses are used to improve focus. Movement of the first and last marker on the slope is recorded by a single camera, but the interior markers (e.g., rows 2-5) are each viewed by two cameras. Videos are converted to a series of images using MATLAB. GEO-PIV was used to process the images to produce displacement time histories (Stanier et al. 2015). Carey et al. (2018b) describe how displacements were converted from pixels to $\mathrm{mm}$ using conversion factors specific to each camera.

Displacements calculated by GEO-PIV analyses were validated by manually counting the number of pixels the surface markers moved during shaking by superimposing images in MATLAB. Figure 13.16 shows an example of this process. In Fig. 13.16a, the location of a typical surface marker prior to and following shaking is overlaid. The dashed yellow box outlines the patch GEO-PIV tracks during shaking to calculate a displacement. Figure 13.16b overlays the same two images as Fig. 13.16a, but the image recorded prior to shaking is vertically shifted until there is a clear image of the surface marker. The pixel offset corresponds to the downslope displacement that marker experienced during shaking.

Figure 13.17 compares downslope displacements found by hand measurement of surface markers with calipers and rulers, GEO-PIV analysis, and manual pixel counting using MATLAB (Fig. 13.16). Surface markers $2-5$ are viewed by two cameras; thus these marker locations have two manual pixel counting measurements. Overall, GEO-PIV was capable of accurately measuring the final displacement of surface markers, arguably better than the physical hand measurements. Table 13.2 provides the differences between GEO-PIV and manual pixel counting measurements were less than about $8 \%$, with the exception of surface marker 1 . The large displacement measurement error for surface marker 1 may be attributed to the fact 


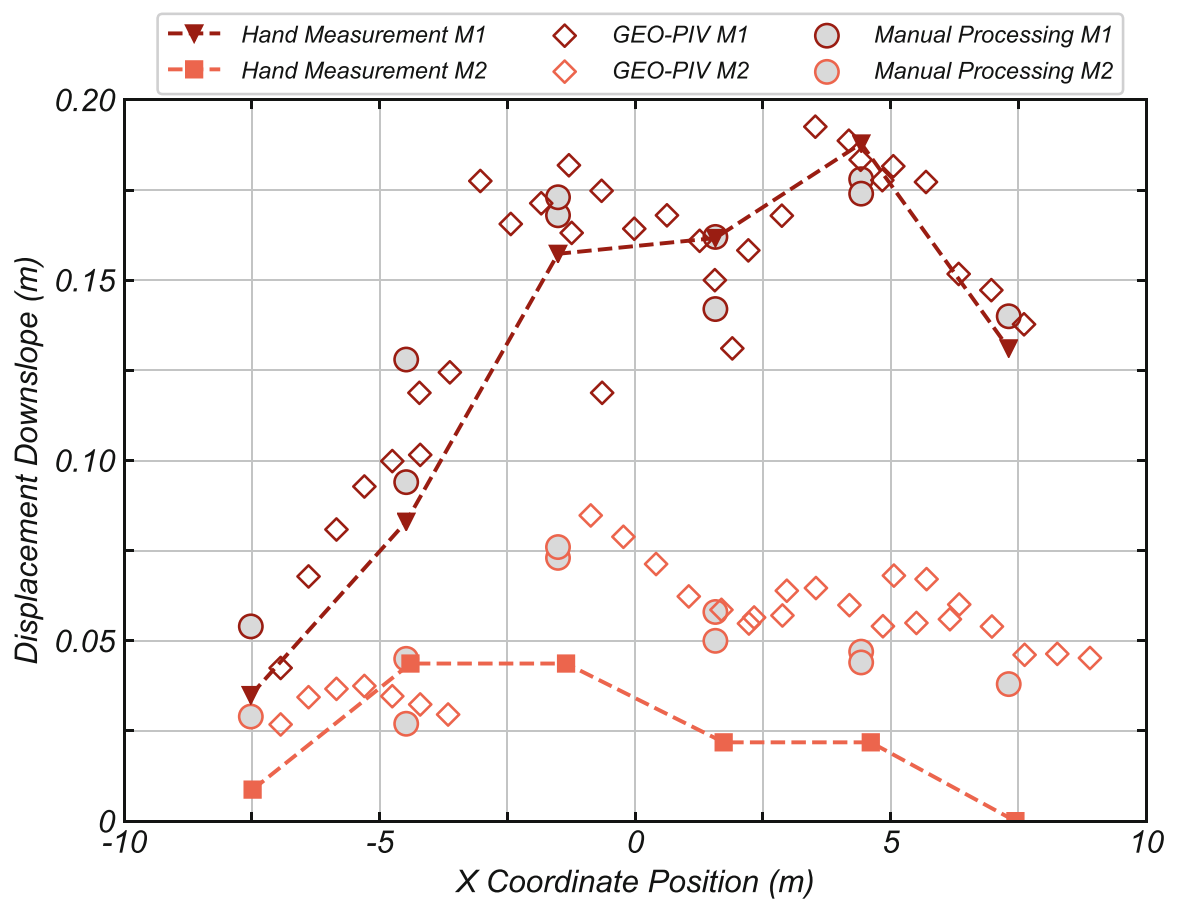

Fig. 13.17 Comparison of surface marker displacement measurements using GEO-PIV, manual image processing in MATLAB, and hand measurements with rulers for centerline surface markers

Table 13.2 Differences in surface marker displacement measurement between GEO-PIV and manual pixel counting

\begin{tabular}{l|l|l}
\hline & Upslope camera & Downslope camera \\
\hline Surface marker 1 & $21.4 \%$ & NA \\
\hline Surface marker 2 & $7.2 \%$ & $8.1 \%$ \\
\hline Surface marker 3 & $1.0 \%$ & $2.0 \%$ \\
\hline Surface marker 4 & $5.6 \%$ & $7.4 \%$ \\
\hline Surface marker 5 & $5.4 \%$ & $3.0 \%$ \\
\hline Surface marker 6 & NA & $1.6 \%$ \\
\hline
\end{tabular}

that surface marker 1 lies only partially in the frame of camera 1 and the GEO-PIV patches do not completely overlap surface marker 1 . An additional benefit of using GEO-PIV to determine downslope displacements is that displacements may be found at more locations because it can track the movement of patches of sand between surface markers.

Figure 13.18 compares displacement time histories generated by GEO-PIV to those found using the IPRV method and accelerometer data, a method to estimate displacement time histories developed by Kutter et al. (2017) and modified by Carey et al. (2018b) for UCD3 M1. The dynamic displacement of the IPRV method is the relative displacement between the base accelerometers and $\mathrm{AH} 4$, found by double 


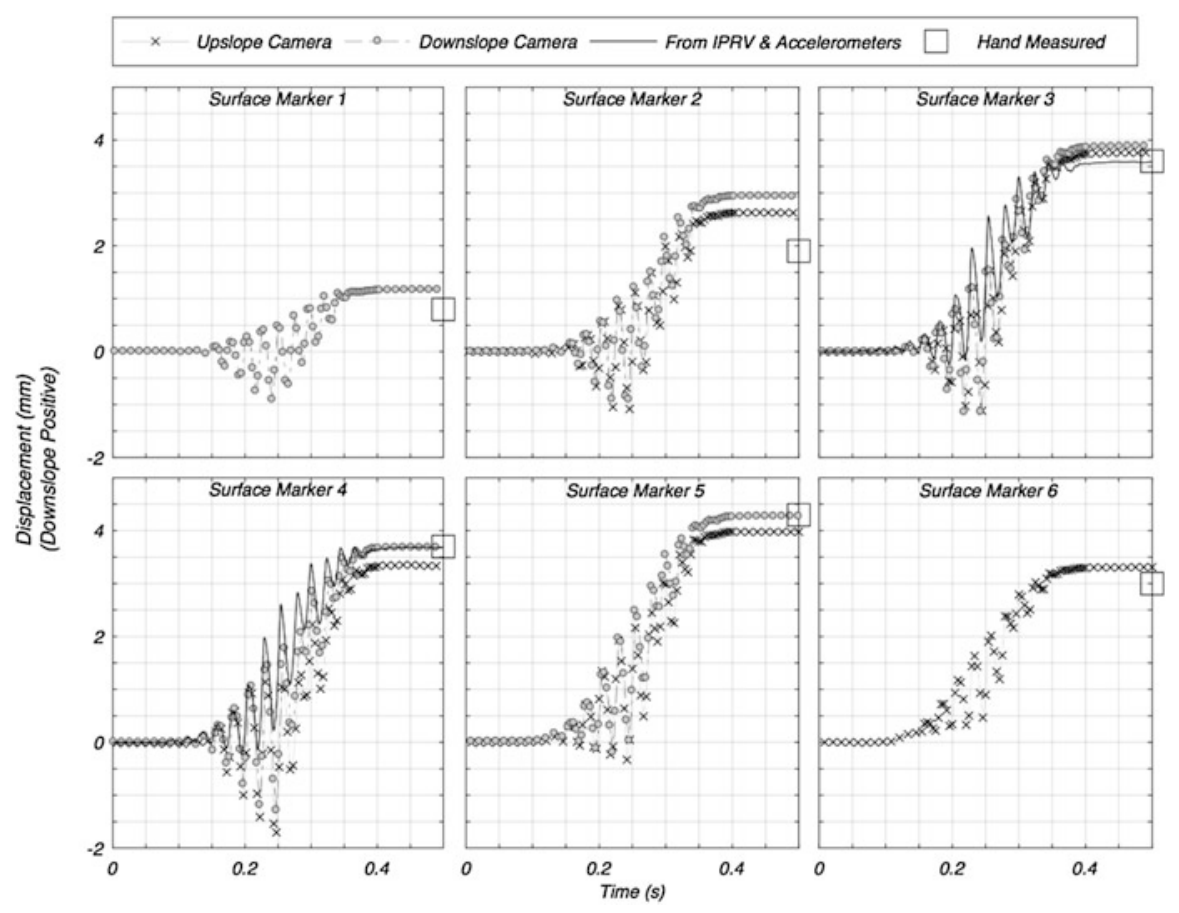

Fig. 13.18 Displacement time series as measured using GEO-PIV and calculated from IPRV method and accelerometer data for UCD3 M1 from Carey et al. 2018b. (Note time and displacement are given in model scale units)

integration of the acceleration at those locations. The final displacement is determined by hand measurement of the surface marker, shown as a square at the end of each time history, and the ramp to the final displacement is the integrated positive relative velocity ramp scaled to match the hand measured marker locations. The IPRV method is only plotted for surface markers 3 and 4 since AH4 is located near those markers. Displacement time histories for markers 2 through 5 are provided for the up- and downslope cameras. Markers 1 and 6 are only recorded by one camera (see Fig. 13.14). Figure 13.18 shows (1) the up- and downslope cameras recorded similar displacement histories for a single marker; (2) the dynamic displacement from GEO-PIV matches well with the dynamic displacement from accelerometers, which are considered to be highly accurate; and (3) the IPRV is a reasonable method to approximate the ramp shape of displacement time histories.

\subsection{Method of Measuring Density}

Density was calculated by mass and volume measurements during model construction as an intermediate check to ensure the target density is achievable. At each intermediate lift, the surface was flattened by vacuuming excess sand and the depth 
Table 13.3 Achieved density of intermediate lifts and final model density

\begin{tabular}{|c|c|c|c|c|c|c|c|c|}
\hline \multicolumn{3}{|l|}{ UCD1 } & \multicolumn{3}{|l|}{ UCD2 } & \multicolumn{3}{|l|}{ UCD3 } \\
\hline $\begin{array}{l}\text { Depth of } \\
\text { lift (mm) }\end{array}$ & $\begin{array}{l}\text { Avg. } \\
\left(\mathrm{kg} / \mathrm{m}^{3}\right)\end{array}$ & $\begin{array}{l}\text { Std } \\
\text { Dev. } \\
\left(\mathrm{kg} / \mathrm{m}^{3}\right)\end{array}$ & $\begin{array}{l}\text { Depth of } \\
\text { lift (mm) }\end{array}$ & $\begin{array}{l}\text { Avg. } \\
\left(\mathrm{kg} / \mathrm{m}^{3}\right)\end{array}$ & $\begin{array}{l}\text { Std } \\
\text { dev. } \\
\left(\mathrm{kg} / \mathrm{m}^{3}\right)\end{array}$ & $\begin{array}{l}\text { Depth of } \\
\text { lift (mm) }\end{array}$ & $\begin{array}{l}\text { Avg. } \\
\left(\mathrm{kg} / \mathrm{m}^{3}\right)\end{array}$ & $\begin{array}{l}\text { Std } \\
\text { dev. } \\
\left(\mathrm{kg} / \mathrm{m}^{3}\right)\end{array}$ \\
\hline 52 & 1693 & 22 & 53 & 1655 & 19 & 57 & 1695 & 13.8 \\
\hline 89 & 1693 & 25 & 115 & 1647 & 6 & 120 & 1659 & 5.96 \\
\hline $\begin{array}{l}\text { Curved } \\
\text { surface } \\
\text { (final } \\
\text { density) }\end{array}$ & 1665 & 6 & $\begin{array}{l}\text { Curved } \\
\text { surface } \\
\text { (final } \\
\text { density) }\end{array}$ & 1648 & 3 & $\begin{array}{l}\text { Curved } \\
\text { surface } \\
\text { (final } \\
\text { density) }\end{array}$ & 1658 & 12.4 \\
\hline
\end{tabular}

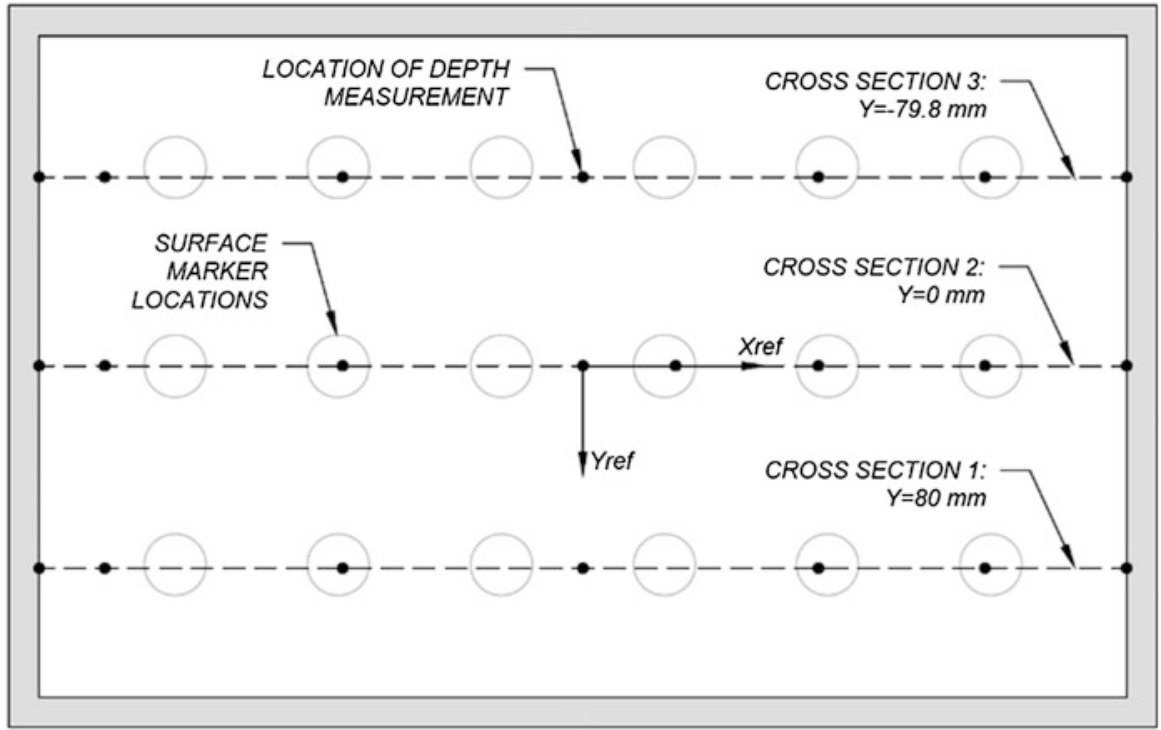

Fig. 13.19 Location of lift height used for volume of model calculation

at 15 locations was measured. The measured depths were averaged, and the density was calculated using the mass of the lift. Reported in Table 13.3 are the depths of the layers measured and the average and standard deviations of the calculated density. The calculation of density for the shallower lifts is more sensitive to variations in lift height. The sensitivity was due to the relatively small mass of the lift and the uncertainty of density was dominated by uncertainty in the lift height.

The reported density of the model used volume and mass measurements of the final curved surface. Figure 13.19 shows the seven locations along the three longitudinal lines where the depth was measured. The three longitudinal lines of measurement roughly corresponded to specified longitudinal lines of the surface markers. Using AutoCAD, a curve in $x-z$ space was fit to the seven points on the 
Area 1: $y=85.3 \mathrm{~mm}$

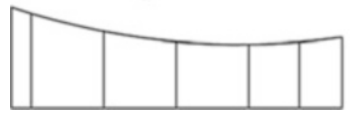

Area 2: $y=0 \mathrm{~mm}$

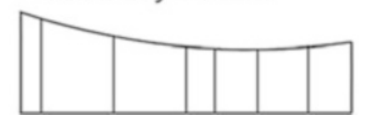

Area 3: $y=-79.8 \mathrm{~mm}$

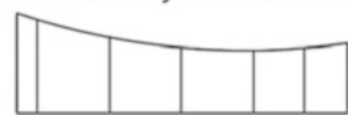

Fig. 13.20 Three cross sections used to calculate the total volume of the completed model with curved surface

same longitudinal line. The MASSPROPS command in AutoCAD was then used to calculate the area of each region, as shown in Fig. 13.20. The volume was estimated by averaging the three areas and multiplying that average by the $279 \mathrm{~mm}$ width of the container.

The load cell used to measure the mass of the model was accurate to about $0.1 \mathrm{~kg}$. The LEAP-UCD-2017 test template contains supplemental documentation of density calculation for each lift during construction of each model.

\subsection{Pore Fluid Viscosity and Saturation}

\subsubsection{Pore Fluid Viscosity}

Methylcellulose was used for each of the UCD experiments to scale the viscosity of the pore fluid in accordance with the scaling law, $\mu=\mu_{\text {water }} / L^{*}$. Prior to centrifuge testing, several batches of viscous solution were mixed to determine the correct proportions of Dow F50 Food Grade methylcellulose powder and water by mass to achieve the desired viscosity of $\mu=43.75$. The ratio of mass of methylcellulose to mass of water was $2.2 \%$.

The methylcellulose was prepared in accordance with the chemical supplier recommendation for hot mixed solution. The procedure was as follows:

1. Warm roughly $1 / 4$ of the required deionized water to $90{ }^{\circ} \mathrm{C}$. Add methylcellulose with a mass that is equal to $8.8 \%$ of mass of the deionized water.

2. Mix solution for $45 \mathrm{~min}$.

3. Dilute the mixture with the same mass of water from step 1 at room temperature. Mix for additional $10 \mathrm{~min}$. Following mixing, this stock solution should be at roughly double the required concentration.

4. Cool overnight.

5. Using an approximately $200 \mathrm{~g}$ sample of the stock solution, $208 \mathrm{~g}$ of room temperature deionized water was added and mixed. Viscosity of the solution was checked using a Cannon instrument size 2 Ubbelohde viscometer.

6. Step 5 was repeated if necessary, adjusting the amount of deionized water added, to determine the ratio of deionized water and stock solution to produce the desired viscosity.

7. Lastly, the entire batch of methylcellulose was mixed with the ratio of water and stock found in Step 6. 


\subsubsection{Model Saturation}

Each of the UCD models followed the same procedure for saturation. Initially, the dry model and container were placed in a vacuum chamber. $97 \mathrm{kPa}$ of vacuum was applied, then the vacuum was shut off, and the chamber was flooded with $\mathrm{CO}_{2}$ up to until the vacuum was reduced to about $2 \mathrm{kPa}$. Then, the $\mathrm{CO}_{2}$ flow was shut off and the $97 \mathrm{kPa}$ vacuum was reapplied. This cycle was repeated two times and following the second cycle the vacuum was reapplied to $97 \mathrm{kPa}$. Following the third evacuation, the residual concentration of nitrogen and oxygen gas in the chamber should be $((101.4-97) / 101.4)^{3}$ times its initial concentration in atmospheric air, and the partial pressure of $\mathrm{CO}_{2}$ gas would be about $4 \%$ of an atmosphere. The saturation chamber and model were inclined so the toe of the model (left-hand side of Fig. 13.1) was the lowest point in the container. The methylcellulose solution was de-aired and dripped on a sponge on the top surface of the sand to maintain a pool of de-aired methylcellulose solution in the lowest edge of the container. As the wetting front progressed toward the top of the model, the size of the pool was allowed to grow. The top corner of the slope was the last location to saturate, allowing for any residual gas and air to escape from the model. Nine-seven $\mathrm{kPa}$ vacuum was maintained throughout the infiltration of methylcellulose. Once the model was completely saturated and the surface submerged, the vacuum was slowly released.

Once the model was saturated, the degree of saturation was then checked using the method described in the specification, modified from Okamura and Inoue (2012) and Carey et al. (2017). The chamber was opened, and a tethered float delineated with a $1 \mathrm{~cm}$ grid was placed on the surface of the methylcellulose. Laser pointers were positioned on the container walls pointing downward at the floating grid with a five-degree angle. The laser pointer and float configuration are shown in Fig. 13.21. The transparent cover was placed back over the vacuum chamber and the locations of the dots from the laser on the float were photographically recorded. A small, $10 \mathrm{kPa}$ vacuum was applied and the location of the laser dots was recorded. Using Boyle's law (Carey et al. 2017) the degree of saturation for all three UCD experiments was calculated to be $99.99 \%$. The tiny $0.01 \%$ bubble volume would easily dissolve in the water with time or with the increased pressure in the centrifuge.

\subsection{Conclusions}

This paper described the three experiments performed on the $1 \mathrm{~m}$ centrifuge at the University of California, Davis, as part of the LEAP-UCD-2017 exercise. The model performance and results for the two destructive motions were presented, and the nonconformities from the specifications were also discussed. The process to mix methylcellulose pore fluid was presented and the protocol to saturate and measure the degree of saturation of the model was also explained.

Motion 1 in UCD1 did not cause liquefaction, which is evident by the low excess pore pressures, and the nearly rigid acceleration response of the model. The second experiment had larger than expected high-frequency component superimposed on the 
Fig. 13.21 Lasers positioned so dots point at float on surface of methylcellulose

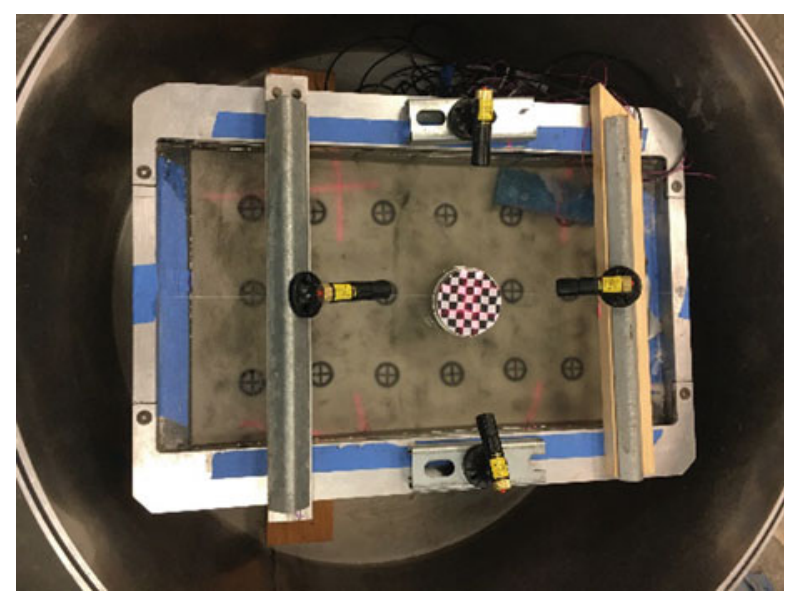

$1 \mathrm{~Hz}$ specified input motion. The achieved velocity closely matched the specified velocity for both motions of the second test. The third experiment showed the best repetition of input motions and model performance, and can be used to determine if numerical simulations correctly model the evolution of soil properties from repeated shaking events. Other minor deviations from the specifications were discussed, such as alternate placement of surface markers, and the use of minor nondestructive ground motions before the destructive motions.

A new technique was presented that uses GoPro cameras mounted on a wave suppressing window acting like a glass-bottomed boat to record images of the deforming surface. GEO-PIV was used to process the images and create displacement time series. Results from the procedure were shown to be reliable when compared against hand measurements, sensor data, and the manual processing of images.

A protocol for measuring the achieved density of a specimen was described. The final density was calculated by measuring the depth of soil along three longitudinal transects and fitting a curve to those depths in AutoCAD. This method to find density, while still relying on point measurements, proved to be helpful for accurately determining the final density and the associated uncertainty of the density measurements.

Acknowledgments These experiments were supported by NSF CMMI Grant Number 1635307. The authors would also like to thank the staff at the Center for Geotechnical Modeling (CGM) for their assistance and technical insight throughout the series of tests.

\section{References}

Carey, T., Gavras, A., Kutter, B., Haigh, S. K., Madabhushi, S. P. G., Okamura, M., Kim, D. S., Ueda, K., Hung, W.-Y., Zhou, Y.-G., Liu, K., Chen, Y.-M., Zeghal, M., Abdoun, T., Escoffier, S., \& Manzari, M. (2018a). A new shared miniature cone penetrometer for centrifuge testing. In Proceedings of 9th International Conference on Physical Modelling in Geotechnics, ICPMG 2018 (Vol. 1, pp. 293-229). London: CRC Press/Balkema. 
Carey, T., Stone, N., Kutter, B., \& Hajialilue-Bonab, M. (2018b). A new procedure for tracking displacements of submerged sloping ground in centrifuge testing. In Proceedings of 9th International Conference on Physical Modelling in Geotechnics, ICPMG 2018 (Vol. 1, pp. 829-834). London: CRC Press/Balkema.

Carey, T. J., Hashimoto, T., Cimini, D., \& Kutter, B. L. (2017). LEAP-GWU-2015 centrifuge test at UC Davis. International Journal of Soil Dynamics and Earthquake Engineering, Elsevier, 113, 663. https://doi.org/10.1016/j.soildyn.2017.01.030.

Fiegel, G. L., \& Kutter, B. L. (1994). Liquefaction mechanism for layered soils. Journal of Geotechnical Engineering, ASCE, 120, 737-755. https://doi.org/10.1061/(asce)0733-9410 (1994)120:4(737).

Kutter, B. L., Carey, T. J., Bonab, M. H., Stone, N., Manzari M., Zeghal M., Escoffier, S., Haigh, S., Madabhushi, G., Hung, W., Kim, D., Kim, N., Okamura, M., Tobita, T., Ueda, K., \& Zhou, Y. (2019a). LEAP-UCD-2017 V. 1.01 model specifications. In B. Kutter et al. (Eds.), Model tests and numerical simulations of liquefaction and lateral spreading: LEAP-UCD-2017. New York: Springer.

Kutter, B. L., Carey, T., Stone, N., Zheng, B. L., Gavras, A., Manzari, M., Zeghal, M., Abdoun, T., Korre, E., Escoffier, S., Haigh, S., Madabhushi, G., Madabhushi, S. S. C, Hung, W.-Y., Liao, T.-W., Kim, D.-S., Kim, S.-N., Ha, J.-G., Kim, N. R., Okamura, M., Sjafuddin, A. N., Tobita, T., Ueda, K., Vargas, R., Zhou, Y.-G., \& Liu, K. (2019b). LEAP-UCD-2017 comparison of centrifuge test results. In B. Kutter et al. (Eds.), Model tests and numerical simulations of liquefaction and lateral spreading: LEAP-UCD-2017. New York: Springer.

Kutter, B. L., Carey, T. J., Hashimoto, T., Zeghal, M., Abdoun, T., Kokkali, P., Madabhushi, S. P. G., Haigh, S. K., Burali d'Arezzo, F., Madabhushi, S. S. C., Hung, W.-Y., Lee, C.-J., Cheng, H.C., Iai, S., Tobita, T., Ashino, T., Ren, J., Zhou, Y.-G., Chen, Y., Sun, Z.-B., \& Manzari, M. T. (2017). LEAP-GWU-2015 experiment specifications, results, and comparisons. International Journal of Soil Dynamics and Earthquake Engineering, Elsevier, 113, 616. https://doi.org/10. 1016/j.soildyn.2017.05.018.

Kutter, B. L., \& Wilson, D. W. (1999). De-liquefaction shock waves. In Proceedings of the seventh US-Japan workshop on earthquake resistant design of lifeline facilities and countermeasures against soil liquefaction, Seattle. pp. 295-310.

Okamura, M., \& Inoue, T. (2012). Preparation of fully saturated models for liquefaction study. International Journal of Physical Modelling in Geotechnics, 12, 39-46. https://doi.org/10.1680/ ijpmg.2012.12.1.39.

Stanier, S. A., Blaber, J., Take, W. A., \& White, D. J. (2015). Improved image-based deformation measurement for geotechnical applications. Canadian Geotechnical Journal, 53, 727-739. https://doi.org/10.1139/cgj-2015-0253.

Open Access This chapter is licensed under the terms of the Creative Commons Attribution 4.0 International Licence (http://creativecommons.org/licenses/by/4.0/), which permits use, sharing, adaptation, distribution and reproduction in any medium or format, as long as you give appropriate credit to the original author(s) and the source, provide a link to the Creative Commons licence and indicate if changes were made.

The images or other third party material in this chapter are included in the chapter's Creative Commons licence, unless indicated otherwise in a credit line to the material. If material is not included in the chapter's Creative Commons licence and your intended use is not permitted by statutory regulation or exceeds the permitted use, you will need to obtain permission directly from the copyright holder.

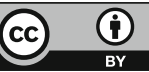

